\title{
Large Harmonic Softening of the Phonon Density of States of Uranium
}

\author{
M. E. Manley, ${ }^{1,2}$ B. Fultz, ${ }^{1}$ R. J. McQueeney, ${ }^{2}$ C. M. Brown, ${ }^{3}$ W. L. Hults, ${ }^{2}$ J. L. Smith, ${ }^{2}$ \\ D. J. Thoma, ${ }^{2}$ R. Osborn, ${ }^{4}$ and J.L. Robertson ${ }^{5}$ \\ ${ }^{1}$ California Institute of Technology, Pasadena, California 91125 \\ ${ }^{2}$ Los Alamos National Laboratory, Los Alamos, New Mexico 87545 \\ ${ }^{3}$ University of Maryland, College Park, Maryland 20742 \\ and National Institue of Standards and Technology, Gaithersburg, Maryland 20899 \\ ${ }^{4}$ Argonne National Laboratory, Argonne, Illinois 60439 \\ ${ }^{5}$ Oak Ridge National Laboratory, Oak Ridge, Tennessee 37831
}

(Received 3 August 2000)

\begin{abstract}
Phonon density-of-states curves were obtained from inelastic neutron scattering spectra from the three crystalline phases of uranium at temperatures from 50 to $1213 \mathrm{~K}$. The $\alpha$-phase showed an unusually large thermal softening of phonon frequencies. Analysis of the vibrational power spectrum showed that this phonon softening originates with the softening of a harmonic solid, as opposed to vibrations in anharmonic potentials. It follows that thermal excitations of electronic states are more significant thermodynamically than are the classical volume effects. For the $\alpha-\beta$ and $\beta-\gamma$ phase transitions, vibrational and electronic entropies were comparable.
\end{abstract}

DOI: 10.1103/PhysRevLett.86.3076

Although first known for its unusual nuclear properties, uranium exhibits several unusual solid-state properties that may originate with electronic instabilities. The thermally induced softening of the phonon density of states (DOS) for most elements originates with anharmonicity $[1,2]$. For the actinides, however, a distinction between the normal anharmonic softening and harmonic softening arising from a temperature-dependent harmonic potential has been suggested [3]. In a detailed assessment of the thermodynamic data on the six crystalline phases of $\mathrm{Pu}$, it was concluded that the anharmonic and electronic contributions to the equation of state could not be separated [4]. The origin of this phonon softening is a fundamental issue for the equation of state. In this Letter, we use the power spectrum of atom motions to show that the thermal softening of the phonon DOS in $\alpha-U$ originates with the weakening of force constants in a harmonic solid, as opposed to the typical softening in an anharmonic potential. Temperature alters the electronic structure sufficiently to change the lattice dynamics. This Letter also addresses the entropy of phonons, and by deduction the entropy of electrons, for the three low-pressure phases of crystalline uranium metal.

Previous lattice dynamics studies on uranium have been performed at room temperature and below [5,6], motivated in part by the discovery of several charge density wave transitions at low temperatures $[7,8]$. Independently, there has been recent interest in the vibrational entropy contribution to the high temperature phase stability of metals and alloys [9-11], motivated by the discovery that vibrational entropy plays a larger role in phase stability than previously expected [12]. Other experimental and theoretical work has shown that electronic contributions to the entropies of high temperature phase transitions can also be significant [13-15].
Diffraction measurements on $\alpha-U$ at ambient pressure have shown that the Debye temperature decreases dramatically with increasing temperature $[3,16]$. This softening is consistent with decreases in the elastic constants $[17,18]$. Specifically, the Debye temperature was expressed by $\theta \cong(306-0.158 T) \mathrm{K}$, where $T$ is temperature [3]. The magnitude of this softening suggests that the Debye temperature decreases by about $40 \%$ between 300 and $940 \mathrm{~K}$. This corresponds to a vibrational entropy of $\Delta S=3 k_{B} \ln \left(\theta_{300 \mathrm{~K}} / \theta_{940 \mathrm{~K}}\right)=1.5 k_{B} /$ atom beyond that of the room temperature phonon DOS.

The usual thermodynamic argument for thermal expansion is that, although thermal expansion generates elastic energy, a less dense crystal has lower phonon frequencies and, hence, a larger vibrational entropy. The quasiharmonic approximation assumes these vibrations to be those of a harmonic solid [1]. In this approximation, the entropy due to phonon softening equals the entropy from volume expansion, consistent with the thermodynamic prediction:

$$
\begin{aligned}
S_{p}-S_{V} & =\int_{300 \mathrm{~K}}^{940 \mathrm{~K}} \frac{C_{p}-C_{V}}{T} d T=9 B_{T} V \alpha^{2}(640 \mathrm{~K}) \\
& =0.16 k_{B} / \text { atom }
\end{aligned}
$$

where the bulk modulus $B=100 \mathrm{GPa}$, the molar volume $V=12.49 \mathrm{~cm}^{3} / \mathrm{mol}$, and the linear thermal expansion coefficient $\alpha=1.39 \times 10^{-5} / \mathrm{K}$ are all values at $300 \mathrm{~K}$ [8]. (Their temperature dependencies are not expected to change the results below by more than a few percent.) The entropy change of $1.5 k_{B}$ /atom predicted with DebyeWaller factors is thus about an order of magnitude larger than what is expected from the volume expansion. This substantial inconsistency suggests that the phonon softening has a different origin. 
All experiments used uranium powder of $99.84 \%$ purity with particle sizes of $20-100 \mu \mathrm{m}$. For safety reasons, the particles were passivated with a uranium dioxide surface layer that made up about $20 \%$ of the total volume. For the high temperature measurements, about $80 \mathrm{~g}$ was loaded into a vanadium can of $1.5 \mathrm{~cm}$ diameter and $7.6 \mathrm{~cm}$ in length. The sample can was mounted in a furnace that was kept under high vacuum throughout all measurements. Neutron energy gain spectra were measured at high temperatures with the Fermi-chopper spectrometer (FCS) at the National Institute of Standards and Technology Center for Neutron Research. The spectrometer was operated with an incident neutron energy of $3.55 \mathrm{meV}(\lambda=4.8 \AA)$. Spectra were obtained on $\alpha-U$ at $300,433,645$, and $913 \mathrm{~K} ; \beta-U$ at $1013 \mathrm{~K}$; and $\gamma-U$ at 1113 and $1213 \mathrm{~K}$. For the low temperature measurements, $157 \mathrm{~g}$ of powder was loaded into a $6 \times 10 \mathrm{~cm}$ flat plate aluminum can of depth $\sim 2.5 \mathrm{~mm}$. The low temperature measurements were performed with the low resolution medium energy chopper spectrometer (LRMECS) at the Intense Pulsed Neutron Source at the Argonne National Laboratory. The spectrometer was operated with incident neutron energies of 25 and $15 \mathrm{meV}$, and measurements were made at 50,250, and $300 \mathrm{~K}$. The data were corrected for empty can scattering and time-independent backgrounds, and summed over a wide range of scattering angles to obtain the phonon DOS. Some weak inelastic intensity from the uranium dioxide surface layer was observed. However, since most of this intensity was at energies higher than the uranium phonon cutoff energy $(\sim 15 \mathrm{meV})$, it was easily fit and subtracted from the elemental uranium scattering using a previously measured phonon DOS of uranium dioxide [19]. The scattering from the oxide accounted for about $10 \%$ of the total inelastic intensity in the energy range of the metallic uranium phonon DOS.

Figure 1 shows the phonon DOS obtained from the measured spectra, corrected for the incoherent multiphonon scattering to all orders in the harmonic approximation using a procedure described elsewhere [14]. The agreement of the FCS and the LRMECS results at $300 \mathrm{~K}$ is encouraging. Both measurements show intensity at $\sim 15 \mathrm{meV}$ above the phonon DOS calculated (DOS_CALC) from the force constant model of Crummett et al. [5]. With this model, the fully coherent one-phonon scattering function, $S_{1}(|Q|, \omega)$, was calculated and summed over the appropriate kinematic $|Q|$ and $\omega$ ranges for each instrument. The results for both LRMECS (LRMECS_CALC) and FCS (FCS_CALC) show that the difference is not a result of insufficient $Q$ sampling. In the $\alpha$ phase, there is a redistribution of intensity in the main features at $\sim 8$ and $\sim 12 \mathrm{meV}$, with the higher energy peak gaining extra weight with increasing temperature. These features also show an overall decrease of about $1 \mathrm{meV}$ per $200 \mathrm{~K}$.

At low temperatures, there is a broadening of the features in the phonon DOS that could arise from shortened phonon lifetimes, perhaps related to the charge density

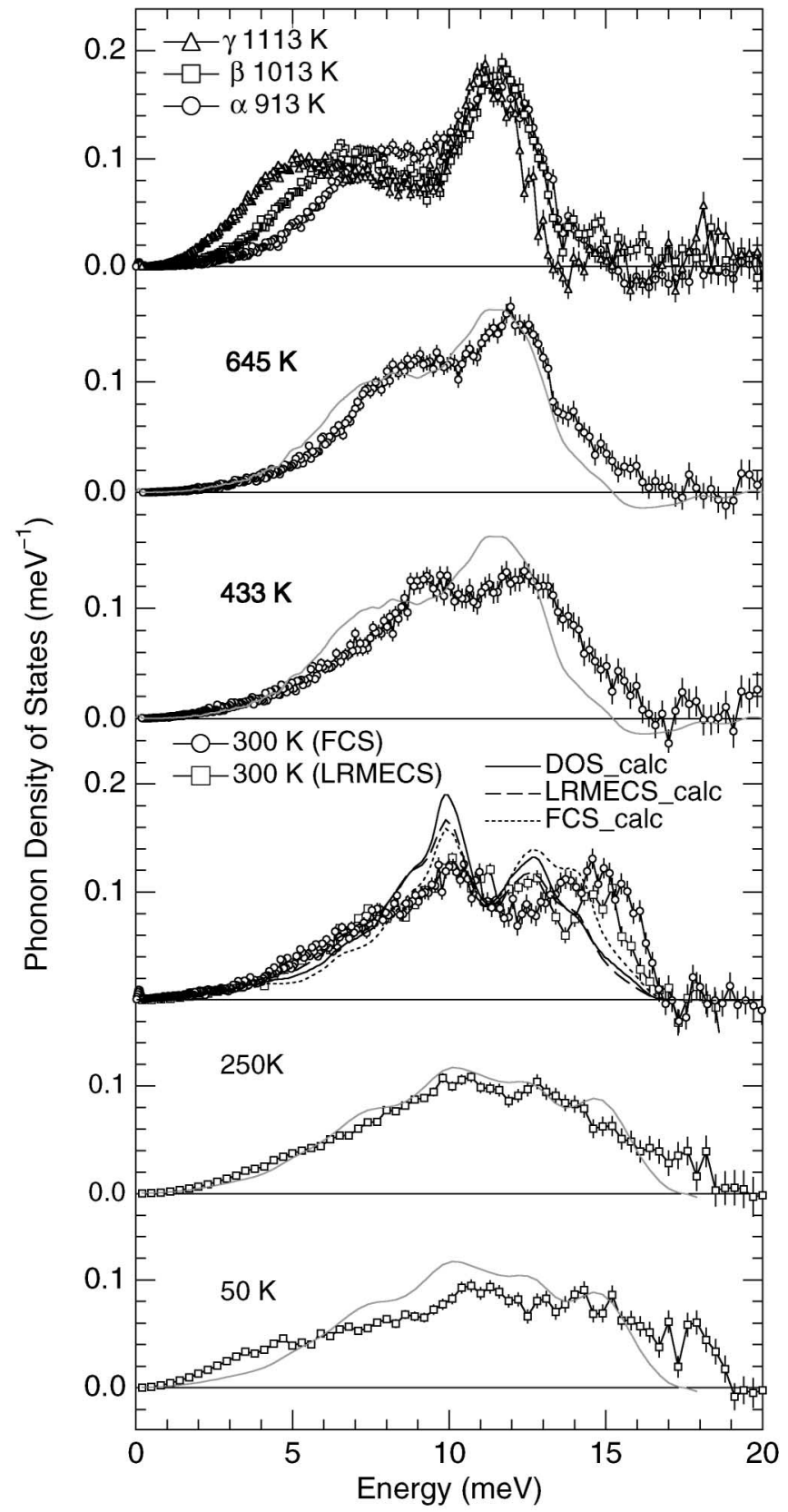

FIG. 1. The phonon DOS of uranium. Data from $300 \mathrm{~K}$ and above were obtained from spectra acquired with Fermi-chopper spectrometer (FCS) at the National Institute of Standards and Technology. Data from $300 \mathrm{~K}$ and below were measured on the low resolution medium energy chopper spectrometer (LRMECS) at Argonne National Laboratory. The curves labeled DOS_CALC, LRMECS_CALC, and FCS_CALC were all calculated from the force constant model of Crummett et al. [5] as described in the text. The $913 \mathrm{~K} \alpha$-uranium DOS is superimposed on all curves above $300 \mathrm{~K}$ and the $300 \mathrm{~K}$ data is superimposed on all curves below $300 \mathrm{~K}$. The three solid state phases, orthorhombic $(\alpha)$, tetragonal $(\beta)$, and body centered cubic $(\gamma)$ are compared at the top.

wave (CDW) transition at $43 \mathrm{~K}$ [8]. At low temperatures, a broad peak appears in the phonon DOS at about $4 \mathrm{meV}$. This could support Yamada's suggestion [20] that a whole sheet of the phonon spectrum starts to soften as the 
temperature is lowered toward the $43 \mathrm{~K} \mathrm{CDW}$ transition a single soft mode would involve a volume in reciprocal space too small to be observed in the phonon DOS.

We used the temperature dependence of the power spectrum to test for harmonic behavior. Equipartition of potential and kinetic energy is expected for harmonic, but not anharmonic, oscillators. For a single component system, the incoherent scattering function, $S_{i}(\boldsymbol{Q}, \omega)$, is given by the Fourier transform of the autocorrelation function [21]:

$$
S_{i}(\boldsymbol{Q}, \omega)=\frac{1}{2 \pi \hbar} \int_{-\infty}^{\infty} \frac{1}{N} \sum_{j=1}^{N}\left\langle e^{-i \boldsymbol{Q} \cdot \boldsymbol{r}_{j}(0)} e^{i \boldsymbol{Q} \cdot \boldsymbol{r}_{j}(t)}\right\rangle e^{-i \omega t} d t,
$$

where $\boldsymbol{r}_{j}$ represents the instantaneous atomic position of atom $j$. By taking the classical limit, the scattering from a crystal in an arbitrary orientation with respect to $\boldsymbol{Q}$ can be simplified by expanding the exponentials in powers of the magnitude of $\boldsymbol{Q}$, denoted $Q$, and integrating over time, $t$, to give

$$
\begin{aligned}
S_{i}(\boldsymbol{Q}, \omega) \cong \frac{1}{N} \sum_{j=1}^{N}( & \frac{\delta(\omega)}{\hbar}\left[1-Q^{2}\left\langle r_{Q, j}^{2}(0)\right\rangle\right] \\
& \left.+\frac{Q^{2}}{2 \hbar}\left|R_{Q, j}(\omega)\right|^{2}+\ldots\right),
\end{aligned}
$$

where $r_{Q, j}$ is the projection of $\boldsymbol{r}_{j}$ along the direction of $\boldsymbol{Q}$, and we have introduced the Fourier transform of $r_{Q, j}(t)$,

$$
R_{Q, j}(\omega)=\int_{-\infty}^{\infty} r_{Q, j}(t) e^{-i \omega t} d t .
$$

For a powder, Eq. (3) is averaged over all orientations. Hence, the modulus square power spectrum averaged over all atoms ( $j$ 's) and directions, denoted $|R(\omega)|^{2}$, can be extracted from the measurement. The average potential energy per degree of freedom can then be determined by integrating the average power spectrum using

$$
\langle U\rangle=\frac{1}{2} M \int \omega^{2}|R(\omega)|^{2} d \omega,
$$

where $M$ is the mass of the vibrating atom. In the case of harmonic phonons in the high temperature limit, the power spectrum is related to the phonon DOS by

$$
\frac{1}{2} M \omega^{2}|R(\omega)|^{2}=\frac{1}{2} k_{B} T Z(\omega) .
$$

Integrating both sides with respect to $\omega$ gives the expected result that $\langle U\rangle_{h}=k_{B} T / 2$. This result holds true even if the harmonic potential is temperature dependent, i.e., if the temperature dependence of the phonon DOS is the result of a continuous change in a harmonic potential. On the other hand, if the potential is constant and the softening originates with anharmonicity of the interatomic potential, the potential energy can be expressed as

$$
\langle U\rangle_{\mathrm{anh}} \cong \frac{1}{2} k_{B} T+A\left(k_{B} T\right)^{2}+B\left(k_{B} T\right)^{3}+\ldots,
$$

where the coefficients $A$ and $B$ can be related to true anharmonic terms in the interatomic potential.

The $Q$-summed one-phonon scattering function, $S_{1}(|Q|, \omega)$, was used with Eqs. (3) and (5) to calculate a quantity proportional to $\langle U\rangle$ for $\alpha-U$ at the four highest temperatures. The result shown in Fig. 2 was scaled so the points at the lowest temperatures were at the harmonic energy $k_{B} T / 2$. Anharmonicity will appear as a nonlinearity in the plot of $\langle U\rangle$ vs $T$. For comparison, attempts were made to calculate the temperature dependence of the potential energy of a Morse and a Lennard-Jones potential. Because the vibrational softening in $\alpha-U$ is so large in spite of its low thermal expansion and elastic stiffness, no standard potential could match all properties. For the potential energy curves labeled "Lennard-Jones" and "Morse_1" in Fig. 2, the correct thermal expansion, nearest-neighbor distance, and vibrational softening $(30 \%)$ were used with an elastic stiffness about 2 times too large. For the potential labeled "Morse_2" in Fig. 2, the correct elastic stiffness was used, but the thermal expansion was about 2 times too large. The nonlinearity in the plot of $\langle U\rangle$ vs $T$ is obviously too large in all cases. The phonon softening in $\alpha-U$ occurs while the potential remains primarily harmonic. Evidently, the interatomic force constants are temperature dependent. Since the force constants originate with the sensitivity of the electronic energy to atom displacements, it follows that thermal excitations of the electronic states are altering the force constants.

The phonon DOS of the three solid-state phases of uranium, orthorhombic $(\alpha)$, tetragonal $(\beta)$, and body centered cubic $(\gamma)$, are compared at the top of Fig. 1. The $\gamma$-uranium phonon DOS was statistically the same at 1113 and $1213 \mathrm{~K}$, showing no evidence of the thermal softening seen in $\alpha$ phase. The $\beta$ phase is not stable over a sufficient temperature range to obtain the temperature dependence of its phonon DOS. The change in phonon DOS at

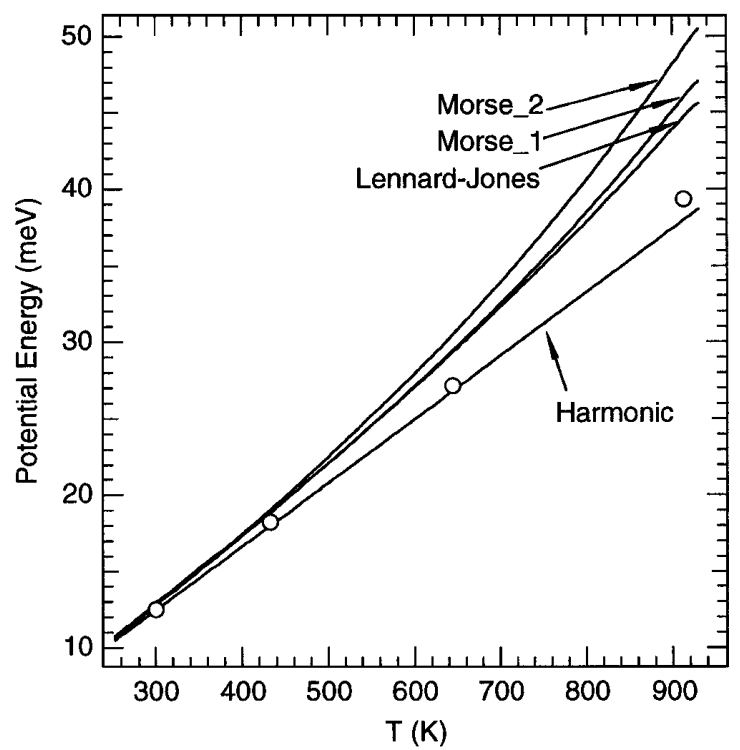

FIG. 2. Vibrational potential energy of $\alpha$ uranium ( $\bigcirc)$. The Lennard-Jones, Morse_1, and Morse_2 curves were calculated from potentials described in the text. The harmonic curve is the result for a harmonic potential in the classical limit. 
each phase transition accounted for vibrational entropy changes of $\left(S^{\beta}-S^{\alpha}\right)_{\text {vib }}=+(0.15 \pm 0.1) k_{B} /$ atom and $\left(S^{\gamma}-S^{\beta}\right)_{\mathrm{vib}}=+(0.36 \pm 0.1) k_{B} /$ atom. The errors arise mainly from the uncertainty in the $Q$ sampling estimated from the difference between the calculated phonon DOS (DOS_CALC) and calculated $Q$-summed coherent scattering for the FCS instrument (FCS_CALC). Both of these values are significantly smaller than the total entropy obtained from latent heat measurements: $\left(S^{\beta}-S^{\alpha}\right)_{\text {tot }}=(0.35-0.37) k_{B} /$ atom and $\left(S^{\gamma}-S^{\beta}\right)_{\text {tot }}=$ $(0.54-0.55) k_{B} /$ atom $[22,23]$. The remaining entropy of the phase transitions must be electronic in origin. Not only does the phonon softening disappear in the high temperature $\gamma$ phase, but it does so with a large increase in electronic entropy. Electronic entropy evidently makes a major contribution to the stability of the $\beta$ and $\gamma$ phases.

The temperature dependence of the electronic structure in $\alpha-U$ plays a major role in its thermodynamics, being comparable to the phonon entropy and overwhelming the usual anharmonic behavior. Present state-of-the-art electronic band structure calculations used to predict properties such as phonon frequencies are based on the assumption that thermal effects on the electronic structure can be neglected when compared to volume effects. The actinides, however, show the need for more sophisticated treatments of the role of temperature on interatomic interactions.

M. E. M. acknowledges discussions with A. C. Lawson, D. A. Neumann, D. A. Dimitrov, and B. R. Cooper. The authors also thank A. M. Kelly for help with the sample characterization. This work was supported by the U.S. Department of Energy under Contracts No. DE-FG0396ER45572 and No. W-31-109-ENG-38.

[1] A. A. Maradudin and A.E. Fein, Phys. Rev. 128, 2589 (1962).

[2] T. H. K. Barron, J. G. Collins, and B. K. White, Adv. Phys. 29, 609 (1980).

[3] A. C. Lawson, B. Martinez, J. A. Roberts, B. I. Bennett, and J. W. Richardson, Jr., Philos. Mag. B 80, 53 (2000).
[4] D. C. Wallace, Phys. Rev. B 58, 15433 (1998).

[5] W. P. Crummett, H. G. Smith, R. M. Nicklow, and N. Wakabayashi, Phys. Rev. B 19, 6028 (1979).

[6] H. G. Smith, N. Wakabayashi, W. P. Crummett, R. R. Nicklow, G. H. Lander, and E. S. Fisher, Phys. Rev. Lett. 44, 1612 (1980).

[7] J. C. Marmeggi and A. Delapalme, Physica (Amsterdam) 120B, 309 (1980).

[8] G. H. Lander, E. S. Fisher, and S. D. Bader, Adv. Phys. 43, 1 (1994).

[9] J. Okamoto, C. Ahn, and B. Fultz, in Proceedings of the XIIth International Congress for Electron Microscopy, edited by L. D. Peachey and D. B. Williams (San Francisco Press, San Francisco, 1990), p. 50.

[10] L. Anthony, J. K. Okamoto, and B. Fultz, Phys. Rev. Lett. 70, 1128 (1993).

[11] L. Anthony, L. J. Nagel, J. K. Okamoto, and B. Fultz, Phys. Rev. Lett. 73, 3034 (1994).

[12] F. Ducastelle, Ordering and Phase Stability in Alloys (North-Holland, Amsterdam, 1991), p. 471.

[13] J. L. Robertson, H. N. Frase, P. Bogdanoff, M. E. Manley, B. Fultz, and R. J. McQueeney, Philos. Mag. Lett. 79, 297 (1999).

[14] M. E. Manley, R. J. McQueeney, J. L. Robertson, B. Fultz, and D. A. Neumann, Philos. Mag. Lett. 80, 591 (2000).

[15] E. G. Moroni, G. Grimvall, and T. Jarlborg, Phys. Rev. Lett. 76, 2758 (1996).

[16] A.C. Lawson, J. A. Goldstone, B. Cort, R. I. Sheldon, and E. M. Foltyn, J. Alloys Compd. 213/214, 426 (1994).

[17] E.S. Fisher, Argonne National Laboratory Report No. ANL-6096, TID-4500, 1960.

[18] E. S. Fisher, J. Nucl. Mater. 18, 39 (1966).

[19] G. Dolling, R. A. Cowley, and A.D. B. Woods, Can. J. Phys. 43, 1397 (1965).

[20] Y. Yamada, Phys. Rev. B 47, 5614 (1993).

[21] G. L. Squires, Introduction to the Theory Neutron Scattering (Dover, New York, 1978), p. 61.

[22] F.L. Oetting, M.H. Rand, and R.J. Ackermann, The Chemical Thermodynamics of Actinide Elements and Compounds, The Actinide Elements (International Atomic Energy Agency, Vienna, 1976), Pt. 1, p. 16.

[23] Rare Metals Handbook, edited by C. A. Hampel (Reinhold, London, 1961), p. 609. 